\title{
Adjusting the voltage step-up ratio of a magnetoelectric composite transformer
}

\author{
LÜ Li $^{1}$, GUO YangYang ${ }^{1}$, ZHOU JianPing ${ }^{1,2 *}$, WANG Pan ${ }^{1}$, LIU Peng ${ }^{1} \&$ CHEN XiaoMing ${ }^{1}$ \\ ${ }^{1}$ College of Physics and Information Technology, Shaanxi Normal University, Xi'an 710062, China; \\ ${ }^{2}$ State Key Laboratory of Electronic Thin Films and Integrated Devices, University of Electronic Science and Technology of China, Chengdu \\ 610054, China
}

Received February 24, 2010; accepted May 20, 2010

\begin{abstract}
A magnetoelectric composite transformer is proposed. The voltage step-up ratio can be adjusted by an applied magnetic field based on the direct and converse magnetoelectric effects. The nonlinear relationship between the voltage step-up ratio and magnetic field is caused by the nonlinear relationship of the magnetoelectric effect in magnetic field.
\end{abstract}

rosen-type transformer, magnetoelectric effect, magnetostriction, piezoelectricity

Citation: $\quad$ Lü L, Guo Y Y, Zhou J P, et al. Adjusting the voltage step-up ratio of a magnetoelectric composite transformer. Chinese Sci Bull, 2011, 56: 700-703, doi: $10.1007 / \mathrm{s} 11434-010-4083-6$

With the development of information technology, materials with good performance and devices with minimal size are highly sought after. The development of new materials and the design of complex composites have become major research topics. Multiferroic materials have attracted increasing interest for their complex properties [1-3]. Magnetoelectric materials exhibit both electric and magnetic properties, and the magnetoelectric effect can be produced by coupling the electric and magnetic properties. Magnetoelectric materials are multifunctional materials, which have potential applications in magnetic sensors, magnetoelectric transducers and data storage devices [4,5]. Many singlephase magnetoelectric materials have weak magnetoelectric coupling and commonly operate at low temperatures [6,7], making them difficult to apply. In contrast, magnetoelectric composites enjoy strong magnetoelectric coupling at room temperature. Moreover, the magnetoelectric coefficients can be adjusted by changing the phase relationships, which is currently attracting the attention of researchers [3]. In recent years, researchers have been optimizing the magnetoelectric output through experimental and theoretical studies. In

*Corresponding author (email: zhoujp@ @nnu.edu.cn) particular, laminated magnetoelectric composites exhibit a very strong magnetoelectric coupling around their resonant frequency [8-12], which has been used as an amplifier [13]. A Rosen-type transformer has enhanced the magnetoelectric coefficient of the composite $[14,15]$. A magnetoelectric device with double output ports was obtained by combining Terfenol-D alloy and a PMN-PT transformer [16]. The key problem is how to use magnetoelectric composites in devices.

The Rosen-type transformer is divided into two basic parts. The input part is polarized across its width and the output part is polarized along its length. When applying an alternating voltage on the input port of the transformer, an amplified voltage was inspired at the output port.

In this study, we propose a composite transformer by combining a magnetostrictive alloy and a Rosen-type transformer. The step-up ratio of the transformer can be adjusted through the direct and converse magnetoelectric effects.

\section{Experimental method}

A magnetoelectric composite transformer was prepared by 
bonding a Terfenol-D slice and a Rosen-type transformer with epoxy bonder. The Rosen-type transformer was bought from Kong Hong New-Materials Ltd, Xi'an, China. The dimensions of the transformer are $28.2 \mathrm{~mm} \times 5 \mathrm{~mm} \times 2 \mathrm{~mm}$ and it is divided into two parts as shown in Figure 1. The ratio of input to output parts of the transformer is $1: 1.13$. Terfenol-D with high magnetostriction was supplied commercially (Baotou Rare Earth Research Institute, China) with dimensions of $27.8 \mathrm{~mm} \times 4.1 \mathrm{~mm} \times 2.51 \mathrm{~mm}$. The composite was cured at $150^{\circ} \mathrm{C}$ for half an hour under $150 \mathrm{MPa}$ to get good mechanical coupling. For comparison purposes, the Terfenol-D was polished to thicknesses of 2.07, 1.77, 1.52 and $1.3 \mathrm{~mm}$.

The voltage step-up ratio of the magnetoelectric composite transformer was defined by the output voltage $\left(V_{\text {out }}\right)$ in response to the input voltage $\left(V_{\text {in }}\right)$, i.e. $t=V_{\text {out }} / V_{\text {in }}$. The sine voltage $V_{\text {in }}$ was applied to the input port of the transformer with a signal generator, and the output voltage $V_{\text {out }}$ was measured using an oscilloscope with a $1 \mathrm{M} \Omega$ resistance load. Resonance occurs when the driving frequency is near the natural frequency of the magnetoelectric composite transformer and a stronger voltage step-up ratio is achieved. Generally, we used a voltage near the resonance frequency, and studied the transformer properties near that frequency. The magnetoelectric composite transformer was put in a magnetic field. The voltage step-up ratio was measured under various magnetic fields applied along the length of the composite.

\section{Results and discussion}

Figure 2(a) shows the frequency dependence of the voltage step-up ratio for the pure Rosen-type transformer. Two resonant peaks appear around $59 \mathrm{kHz}$ and $118 \mathrm{kHz}$, corresponding to the half- and full-wavelength resonant modes. The relative voltage step-up ratio is 75 and 115. Figure 2(b) shows the frequency dependence of the voltage step-up ratio under various magnetic fields for the magnetoelectric com-

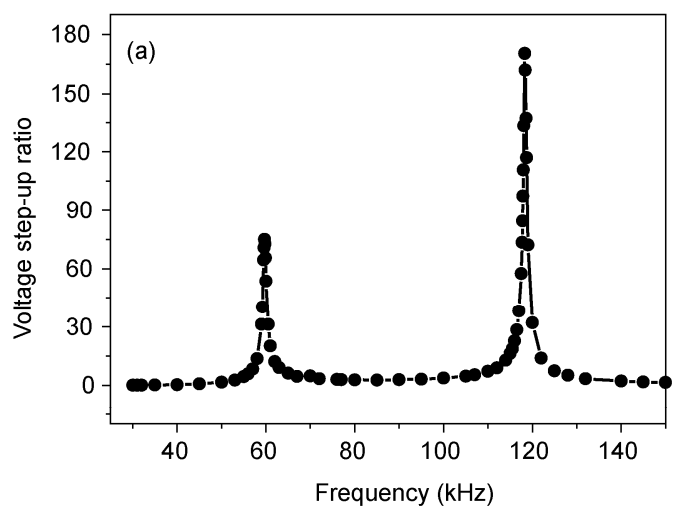

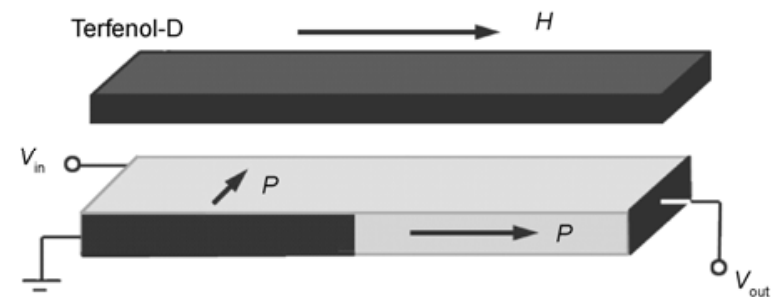

Figure 1 Illustration of the magnetoelectric composite transformer. The magnetic field was applied along the length of the transformer.

posite transformer with a Terfenol-D thickness of $2.51 \mathrm{~mm}$. Similarly, two large sharp peaks of voltage step-up ratios appear at $51 \mathrm{kHz}$ and $101 \mathrm{kHz}$, representing the half- and full-wavelength vibration modes. The step-up ratio without an external magnetic field is 3.1 and 4.2 for the half- and full-wavelength modes. In comparison with the pure PZT transformer, the step-up ratio decreased due to the load of the Terfenol-D plate. After applying the magnetic field, the step-up ratio clearly changed. The step-up ratio peaks decreased and then increased with the magnetic field, while the resonant frequency increased, which was due to the different acoustic velocities of the magnetostrictive Terfenol-D under different magnetic fields [15,17].

The step-up ratios at half- and full-wavelength resonant frequencies share similar characteristics. The Rosen-type transformer commonly works at the full-wavelength vibration mode. Therefore, we studied the step-up ratio characteristics with the magnetic field in the full-wavelength vibration mode. As shown in Figure 3, the step-up ratios with different Terfenol-D thicknesses share similar characteristics with the bias magnetic field. The step-up ratios increase at first and then decrease with increasing magnetic field. Without an external magnetic field, the step-up ratio for the sample with a Terfenol-D thickness of $1.33 \mathrm{~mm}$ is 7.7 . It changes to 4.2 at $250 \mathrm{Oe}$ and 9.2 at 2500 Oe. The step-up ratio nearly doubles over the magnetic field range of 0-250 Oe. Therefore, we can adjust the step-up ratio by changing the magnetic field. The step-up ratio, under the same magnetic field, increases with decreasing Terfenol-D thickness

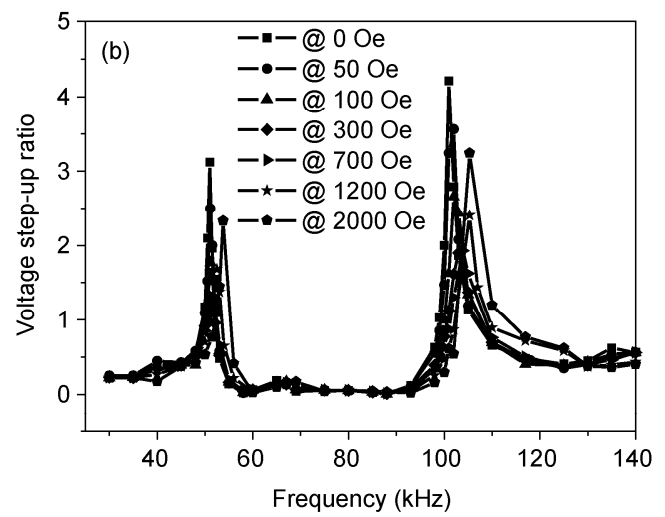

Figure 2 Frequency dependence of the voltage step-up ratio of (a) a pure Rosen-type transformer and (b) a magnetoelectric composite transformer under selected magnetic fields. 
from 2.51 to $1.3 \mathrm{~mm}$. The step-up ratio, without a magnetic field, is 4.7 for a Terfenol-D thickness of $2.51 \mathrm{~mm}$, and 7.6 for $1.3 \mathrm{~mm}$. The increase nearly doubles. This is because the Terfenol-D is equivalent to a load on the transformer. The smaller load results in a higher step-up ratio. Relatively, the resonant frequency increases with a near linear relationship to the magnetic field except at the low magnetic field levels, which is caused by the complex magnetoelectric properties near the resonant frequency [17].

The combining of the direct and converse magnetoelectric effects caused the step-up ratio of the magnetoelectric composite transformer to be adjusted by the magnetic field. After applying an $\mathrm{AC}$ voltage on the input port of the transformer, an amplified signal was produced due to the amplifying effect. At the same time, magnetization is induced due to the converse magnetoelectric effect. The magnetization produces a voltage at the output port due to the direct magnetoelectric effect, which is superimposed on the amplified signal. Therefore, the step-up ratio of the transformer can be adjusted by the magnetic field because the magnetoelectric coupling varies under different magnetic fields.

The magnetization was induced with the piezoelectric material polarized across the width and the magnetostrictive material magnetized along the length. Thus, the converse magnetoelectric coefficient is [22]

$$
\left.\frac{\mathrm{d} H_{3}}{\mathrm{~d} E}\right|_{T-L}=\frac{n(1-n) d_{33, m} d_{31, p}}{\mu_{33}^{T}\left[(1-n) s_{33}^{H}\left(1-k_{33, m}^{2}\right)+n s_{11}^{E}\right]},
$$

where $n$ is the geometric thickness ratio of the piezoelectric layer to the total thickness of the laminate; $s_{11}^{E}$ and $s_{33}^{H}$ are the elastic compliances of the piezoelectric and magnetostrictive layers; $k_{31, m}$ and $\mu_{33}^{T}$ are the electromechanical coefficient and magnetic permeability of piezomagnetic phase under stress; $d_{31, p}$ and $d_{31, m}$ are the piezoelectric and piezomagnetic coefficients. When both the magnetic field and polarized directions are along the length, the direct

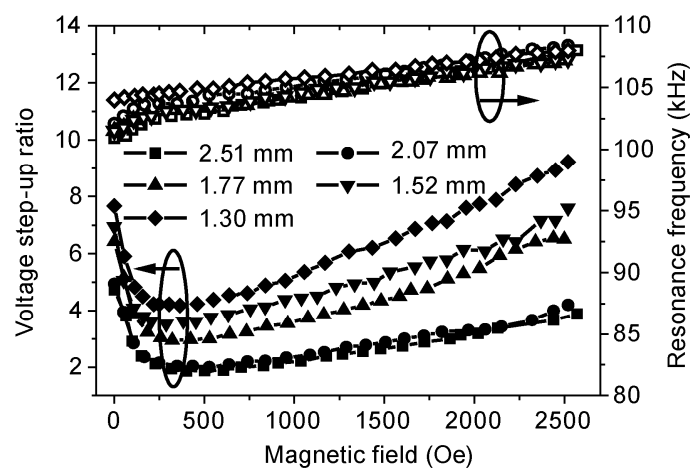

Figure 3 Variation of the voltage step-up ratio of the sample and the resonant frequency with magnetic fields for different thicknesses of the Terfenol-D layer. magnetoelectric coefficient is [22,23]

$$
\left.\frac{\mathrm{d} E}{\mathrm{~d} H_{3}}\right|_{L-L}=\frac{n(1-n) d_{33, m} d_{33, p}}{\varepsilon_{33}^{E}\left[n s_{33}^{E}\left(1-k_{33, p}^{2}\right)+(1-n) s_{33}^{H}\right]},
$$

where $s_{33}^{E}$ and $s_{33}^{H}$ are the elastic compliances of the piezoelectric and magnetostrictive layers; $k_{31, p}$ and $\varepsilon_{33}^{E}$ are the electromechanical coefficient and dielectric constant of piezoelectric phase.

Similar to the permittivity and magnetic permeability, the two parameters of the magnetoelectric modulus and phasic difference have been used to describe the complete magnetoelectric characteristics in the experiments $[8,9,15,20]$. However, while the magnetoelectric modulus was discussed in theory, the phasic difference was not covered [22-25]. In this study, both the magnetoelectric modulus and phasic difference are important because the magnetoelectric voltage superimposes on the amplifying signal. $d_{31, p}$ and $d_{33, p}$ have opposite signs. There are anti-phasic differences between the input voltage and the voltage produced through the converse and direct magnetoelectric effects. Thus, the output voltage reduces after the superimposition of the magnetoelectric signal; as a result, the voltage step-up ratio is reduced as shown in Figure 3. Therefore, stronger magnetoelectric coupling results in a smaller voltage step-up ratio.

The direct and converse magnetoelectric effects share the same characteristics with the increase in the bias magnetic field, i.e., increase and then decrease [8,17-21]. The magnetic field corresponding to the maximum magnetoelectric coupling relates to the demagnetization factor or the shape of the magnetic layer. The maximum magnetoelectric coupling is at about 250 Oe for the Terfenol-D shape in this study [17]. Therefore, the minimal step-up ratio occurs at a magnetic field of 250 Oe.

\section{Conclusions}

In summary, a magnetoelectric composite transformer was prepared by combining a Terfenol-D slice and a Rosen-type transformer. The voltage step-up ratio of the transformer was adjusted by changing the bias magnetic field based on the direct and converse magnetoelectric effects. The dependence of the voltage step-up ratio on the magnetic field shared similar behavior to transformers with different Terfenol-D thicknesses, i.e., decreased at first and then increased with the increase in the magnetic field. The magnetoelectric composite transformer provides a method for the application of magnetoelectric materials.

This work was supported by the National Natural Science Foundation of China (50772065, 50972088) and Open Fund of State Key Laboratory of Electronic Thin Films and Integrated Devices, UESTC (KFJJ200809). 
1 Eerenstein W, Mathur N D, Scott J F. Multiferroic and magnetoelectric materials. Nature, 2006, 442: 759-765

2 Ramesh R, Spaldin N A. Multiferroics: Progress and prospects in thin films. Nat Mater, 2007, 6: 21-29

3 Nan C W, Bichurin M I, Dong S, et al. Multiferroic magnetoelectric composites: Historical perspective, status, and future directions. J Appl Phys, 2008, 103: 031101-1-35

4 Shi Z, Wang C P, Liu X J, et al. A four-state memory cell based on magnetoelectric composite. Chinese Sci Bull, 2008, 53: 1177-1179

5 Bibes M, Barthélémy A. Multiferroics: Towards a magnetoelectric memory. Nat Mater, 2008, 7: 425

6 Wang K M, Liu J M, Wang Y. Single phase multiferroic materials__coupling and adjusting between polar and magnetic order parameters (in Chinese). Chinese Sci Bull, 2008, 53: 1098-1135

7 Chi Z-h, Jin C-q, Recent advances in single-phase magnetoelectric multiferroic(ferroelectromagnet) (in Chinese). Prog Phy, 2007, 27: 225-238

8 Zhou J P, Zhao W, Guo Y Y, et al. Magnetoelectric coupling in small $\mathrm{Pb}(\mathrm{Zr}, \mathrm{Ti}) \mathrm{O}_{3}$ /terfenol-D laminate composites. J Appl Phys, 2009, 105: 063913

9 Shi Z, Ma J, Lin Y, et al. Magnetoelectric resonance behavior of simple bilayered $\mathrm{Pb}(\mathrm{Zr}, \mathrm{Ti}) \mathrm{O}_{3}-(\mathrm{Tb}, \mathrm{Dy}) \mathrm{Fe}_{2}$ /epoxy composites. J Appl Phys, 2007, 101: 043902

10 Dong S, Zhai J, Xing Z, et al. Giant magnetoelectric effect (under a dc magnetic bias of 2Oe) in laminate composites of FeBSiC alloy ribbons and $\mathrm{Pb}\left(\mathrm{Zn}_{1 / 3}, \mathrm{Nb}_{2 / 3}\right) \mathrm{O}_{3}-7 \% \mathrm{PbTiO}_{3}$ fibers. Appl Phys Lett, 2007, 91: 022915

11 Zhai J, Li J,Viehland D, et al. Large magnetoelectric susceptibility: The fundamental property of piezoelectric and magnetostrictive laminated composites. J Appl Phys, 2007, 101: 014102

12 Wan J G, Li Z Y, Wang Y, et al. Strong flexural resonant magnetoelectric effect in Terfenol-D/epoxy- $\mathrm{Pb}(\mathrm{Zr}, \mathrm{Ti}) \mathrm{O}_{3}$ bilayer. Appl Phys Lett, 2005, 86: 202504

13 Dong S, Li J F, Viehland D, et al. A strong magnetoelectric voltage gain effect in magnetostrictive-piezoelectric composite. Appl Phys Lett, 2004, 85: 3534-3536

14 Jia Y M, Luo H S, Zhao X Y, et al. Giant magnetoelectric response from a piezoelectric/magnetostrictive laminated composite combined with a piezoelectric transformer. Adv Mater, 2008, 20: 4776-4779

15 Wang Y, Wang F, Or S W, et al. Giant sharp converse magnetoelectric effect from the combination of a piezoelectric transformer with a piezoelectric/magnetostrictive laminated composite. Appl Phys Lett, 2008, 93: 113503

16 Wang Y, Leung C M, Wang F, et al. Dual-mode magnetoelectric effect in laminate composite of Terfenol-D alloy and PMN-PT transformer with double output ports. J Phys D: Appl Phys, 2009, 42: 135414

17 Guo Y Y, Zhou J P, Liu P, Magnetoelectric characteristics around resonance frequency under magnetic field in $\mathrm{Pb}(\mathrm{Zr}, \mathrm{Ti}) \mathrm{O}_{3} /$ Terfenol-D laminate composite. Curr Appl Phys, 2010, 10: 1092-1095

18 Fetisov Y K, Kamentsev K E, Chashin D V, et al. Converse magnetoelectric effects in a galfenol and lead zirconate titanate bilayer. $\mathrm{J}$ Appl Phys, 2009, 105: 123918

19 Peng S, Yang P, Cai W, et al. Magnetoelectric study in Terfenol-D/ $\mathrm{Tb}_{2}\left(\mathrm{MoO}_{4}\right)_{3}$ bilayer composite. J Appl Phys, 2009, 105: 061622

20 Li L, Chen X M. Magnetoelectric characteristics of a dual-mode magnetostrictive/piezoelectric bilayered composite. Appl Phys Lett, 2008, 92: 072903

21 Chen S Y, Wang D H, Han Z D, et al. Converse magnetoelectric effect in ferromagnetic shape memory alloy/piezoelectric laminate. Appl Phys Lett, 2009, 95: 022501

22 Jia Y M, Luo H S, Or Siu Wing, et al. Magnetoelectric and converse magnetoelectric responses in $\mathrm{Tb}_{x} \mathrm{Dy}_{1-x} \mathrm{Fe}_{2-y}$ alloy \& $\mathrm{Pb}\left(\mathrm{Mg}_{1 / 3} \mathrm{Nb}_{2 / 3}\right)_{1-x} \mathrm{Ti}_{\mathrm{x}} \mathrm{O}_{3}$ crystal laminated composites, Chinese Sci Bull, 2008, 53: 1172-1176

23 Yang F, Wen Y M, Li P, et al. The resonant magnetoelectric response of magnetostrictive/piezoelectric laminated composite under the consideration of losses. Acta Phys Sin-Ch Ed, 2007, 56: 3539-3545

24 Petrov V M, Srinivasan G, Bichurin M I, et al. Theory of magnetoelectric effect for bending modes in magnetostrictive-piezoelectric bilayers. J Appl Phys, 2009, 105: 063911

25 Dong S, Li J-F, Viehland D, Longitudinal and transverse magnetoelectric voltage coefficients of magnetostrictive/piezoelectric laminate composite: Theory. IEEE Trans Ultrason Ferroelectr Freq Control, 2003, 50: 1253-1261

Open Access This article is distributed under the terms of the Creative Commons Attribution License which permits any use, distribution, and reproduction in any medium, provided the original author(s) and source are credited. 